\title{
Chapter 1 \\ Scanning and Scoping of Values and Valuing in Mathematics Education
}

\author{
Philip Clarkson, Wee Tiong Seah and JeongSuk Pang
}

\begin{abstract}
Although the ideas of values and valuing have been totemic notions in education for forever, when applied to mathematics they become quite problematic. Even today for many mathematic teachers and learners, mathematics is a value-free space. For them, school mathematics is learning the skills of manipulating numbers before moving to the more abstract ideas of algebra, and occasionally delving into geometry and measurement ideas. Likewise teaching mathematics in schools is ensuring students get good marks on the tests and examinations using whatever pedagogical techniques ensure this. Although in schools this is still the prevailing attitude to mathematics, nevertheless for some decades there has been a growing counter position in mathematics education research that problematizes and challenges this orthodoxy. It has argued that at a fundamental level there are mathematical values that underpin the doing of mathematics, and indeed the same is true for mathematics pedagogy. This chapter briefly explores a number of these notions as it introduces the various chapters in this volume.
\end{abstract}

Keywords Values $\cdot$ Valuing $\cdot$ Mathematics education $\cdot$ Mathematics • Mathematics teaching $\cdot$ Mathematics learning

\author{
P. Clarkson $(\varangle)$ \\ Australian Catholic University, Locked Bag 4115, Fitzroy MDC, VIC 3065, Australia \\ e-mail: Philip.Clarkson@acu.edu.au \\ W. T. Seah \\ The University of Melbourne, Melbourne, VIC, Australia \\ e-mail:wt.seah@unimelb.edu.au \\ J. Pang \\ Korea National University of Education, Cheongju, South Korea \\ e-mail: jeongsuk@knue.ac.kr




\subsection{Introduction}

Values and valuing, particularly in the context of doing, teaching and learning school mathematics, is a confusing, complex, and contested terrain where colleagues have struggled to even find common definitions of values and valuing, although many have nuanced each other's ideas. This has led to an area of research that many shy away from since it seems that no one really knows what is of worth in the discussion. And yet clearly the notions of values and valuing have been at the heart of education ever since it was, and hence must have a role in researching, teaching and learning mathematics.

So, even though there is ferment in this discussion, over the years the debate regarding values and valuing and mathematics education has provided fertile intellectual opportunities for some scholars to tweak, critique, alter, expand, deconstruct, or devise their own particular standpoints. One way to explore this crucial area of scholarship is to note the contributions from various colleagues who have influenced our thinking.

Among the most influential in the context of mathematics education for many of us has been the contributions of Alan Bishop, to whom this book is dedicated. Bishop has always recognized the importance of the interaction of the social and political within mathematics education, but during his three months sabbatical visit with Glen Lean at the Papua New Guinea University of Technology in 1977 these notions crystalized into an imperative in his research. Mainly from that experience he developed his seminal book on mathematical enculturation (Bishop 1988) where among other ideas he formulated his notion of three pairs of complementary mathematical values and how they are, whether we recognize this or not, a critical influence on how we think about teaching mathematics and how students learn mathematics (Clarkson and Presmeg 2008). Bishop has written extensively on this theme ever since, and many authors in this volume use these six values as either a starting point for their research, or nuancing them, or in other ways reference them.

In the last decade or so, two main thrusts of researching values and valuing in mathematics pedagogy could be identified. The first is made up of different research studies conducted by Turkish researchers based at a number of Turkish universities. These include Büşra Kirez, Esra Selcen Yakıc1-Topbaş, Fatma Nur Aktaş, Gülcin TanŞişman, Nesrin Özsoy, Yüksel Dede, and many others. Amongst them, Yüksel Dede's attachment in Germany had also facilitated comparative studies between German and Turkish students and teachers (see, for example, Chap. 10 in this book). Some of these comparative studies were also conducted as part of Turkish participation in the second thrust of values research in mathematics education.

Unlike the Turkish research focus which represents the first thrust, the second thrust was not country-specific. Called the 'Third Wave Project', it is a series of values research studies conducted by as many as 23 research teams based in 20 different economies, and coordinated by Wee Tiong Seah who is based in Australia. Each study in the Third Wave Project would typically be conducted in different education systems across the world, thus producing findings at the levels of both 
individual economies and across economies. Amongst these, the largest scale and arguably the most well-known study would be the 'What I Find Important (in my mathematics learning)' study, commonly called the WIFI Study. More than 18,000 student questionnaires have been collected and analysed. WIFI data can be seen in Chaps. 6 (Ghana) and 13 (mainland China) in this book. It is important to note that for most of the participating economies such as Australia, Ghana and Japan, the coordinating institutions subsequently lead ongoing research efforts into values in mathematics education within their respective economies.

Although Bishop (1988) had conceptualised three pairs of complementary mathematical values, that is, values which characterise the nature of mathematics as it is presented in classrooms in the 'West', empirical studies that had been conducted since have not identified any other mathematical value to add to this list. At the same time, research studies have also identified different mathematics educational and general educational values, using Bishop's (1996) categorisations, as we will see in the rest of this book. These seem to span across a large range of possibilities, however.

\subsection{Formation of the Book}

This volume is a contribution to the book series that had its foundation at the ICME13 conference (Kaiser 2017), held in 2016. During that conference two of the editors, Clarkson and Seah (with Alan Bishop, Penelope Kalogeropoulos and Annica Andersson), led a Discussion Group entitled Connections Between Valuing and Values: Exploring Experiences and Rethinking Data Generating Methods (Clarkson et al. 2017). During the first of two sessions that the Discussion Group held, we had a number of contributions that discussed where the study of values and valuing in mathematics education had come from and what was happening around the world at that time in various studies. Two key references for this discussion were a special issue of ZDM Mathematics Education (Seah and Wong 2012), and an article that some of us had written (Seah et al. 2016). In the second session of the Discussion Group we directed a role play, based on an earlier version which three of us had run previously at the 35th PME conference held in 2015 (Clarkson 2015), which further explored the mathematical values that Bishop had written about. This present volume extends further the discussions we held during session one of the ICME Discussion Group, and through the agency of the role-play were extended in the second session. Our call for chapters for this volume was broadcast as follows:

Despite the money and time that have been invested over the last few decades in mathematics educational research, improvements of the learning and teaching of mathematics in schools does not appear to have kept pace. For example, although there is some variation between countries, overall student misconceptions of various mathematical concepts remain the same, student engagement in mathematics learning 
has remained low, and we do not seem to be able to improve student attitudes towards mathematics learning.

Reasoning and feelings are clearly part of the learning and teaching of mathematics. But also involved are students' interaction with their cultural setting and each other. The relative recent development of the socio-cultural approaches to understanding and facilitating mathematics education research has complemented the more traditional cognitive and affective traditions. But added to all of these the construct of values has been a promising and useful notion. The significance of values and valuing in other fields of studies, such as science, medicine and business is well established. However values and valuing in the context of mathematics learning and teaching has only been explored from the mid 1980s.

This volume will bring together some of the world's leaders in this aspect of mathematics educational research, who will be reporting on the latest academic knowledge of a chosen theme (e.g. engagement, special education) from the values perspective, discussing how a values/valuing perspective can better facilitate a more effective mathematics pedagogical experience, and proposing implications for research and classroom practice relevant to the them. Reflecting the socio-cultural nature of the values construct, this volume will also feature the intellectual work of researchers from different ethnicities and nationalities. As a collective whole, this should stimulate the reader to further consider each of the featured themes in cross-cultural ways.

The intention of this volume then will focus on conceptual aspects, in terms of how values and valuing play a role in complementing cognition and affect in mathematics learning and teaching. In due course we hope to edit a second volume that will extend this discussion by focusing more on the practical, intervention aspects of values in mathematics education.

\subsection{Chapter Outlines}

We have not tried to squeeze the chapters for this volume into set sections. Although there are some obvious overlap between some chapters over and above them dealing in some way with values and valuing and mathematics, there are also some isolates. In any case the overlaps that we as editors may see might not capture important synergies, and indeed might obscure other overlaps that could be crucial to the thinking of some readers. Hence we have thought about this set of chapters more like small streams that have gradually coalesced into a larger flow, but we leave it to you the reader to decide when the various convergences happen.

With these thoughts in mind, and noting that the order of such is somewhat random, we turn now to introduce the various chapters in this volume.

Clarkson's chapter documents the recorded conversation between himself and Alan Bishop - arguably the father of research into values and valuing in mathematics education-just prior to Alan's return to UK after 25 years in Australia. As would be expected, much was discussed, which included reflections about two projects they 
co-led; the 'Values and Mathematics Project (VAMP)' and as the 'Mathematical Well-being construct' project. There is also a beautiful analogy of mathematics as weaving, with the warps representing the values that are inherent in the discipline. The main focus of the chapter, however, is on the three pairs of complementary mathematical values, which Bishop (1988) conceptualised. The much-talked about question over the years, 'should there be only six mathematical values?' was also touched on. Many research questions were raised throughout the conversation, either explicitly or otherwise, and these should provide many researchers with stimuli for formulating research in this aspect of mathematics education. Of course, the answers to some of these questions might be found in the other chapters of this book (e.g. 'changes in students' values' is examined in Dede's chapter), and the keen reader will no doubt delight in making the connections as s/he peruses the entire book. Delightfully, the chapter ends with a provocative sentence; 'one hopes the reader will also be challenged to think broadly on the notion of mathematical values, a crucial element of the foundational frame that holds what we understand as western mathematics'. This has the look and feel of a cliff-hanger ending to a movie, with a promise of a sequel to come! So, we are reminded of the existence of other kinds of mathematics, and thus, possibly other categories or types of values as well. It does look like there remains much more to what we currently understand and know of values and valuing in the context of mathematics education.

Carr presents an updated systematic literature review of values and valuing in mathematics education with a data set of 34 empirical studies published in peerreviewed journals from 2003 onward. She provides us with a brief but concise summary of all the studies. In order to identify what has been achieved in this field, Carr explores where the research has been conducted, which stakeholders (e.g., teacher, student) are represented, what has been known regarding the development of values, and how consistent the findings of the research are. On the basis of the research findings, Carr suggests the evolving definition of values is a reflection of motivation and effort. She recommends that future research be carried out in respect to the role of conation in shaping values, the relationship between valuing of achievement and academic performance outcome, and changes in values in mathematics teaching and learning. She also calls for studies that go beyond one-off self-report questionnaires.

Corey and Ninomiya focus on teachers and the values they displayed when planning to teach mathematics, and when doing the actual teaching of mathematics. The authors discuss particular community values that Japanese teachers bring to their craft: writing detailed lesson plans, kyozaikenkyu (a planning practice), and emphasizing student mathematical reasoning in instruction. From their analysis they found eight specific values that seem to be essential to the Japanese mathematics teaching community. The study reported by the authors is part of a much larger longitudinal cross cultural study in Japan and the USA and they note some quite interesting contrasts and surprising similarities between these Japanese teachers and their USA counterparts. A number of the values identified here that are important to Japanese teachers clearly informed the notions of lesson study. It is probable that the implementation of lesson study might not be as efficacious elsewhere unless the non Japanese implementing teachers hold the same or similar values. 
Andersson and Österling are concerned with the dilemma of conflicting values between the democratic actions intended in the Swedish curriculum and the most valued mathematical activities nominated by Swedish students. An active participation by students in mathematics classrooms has been politically emphasized, which the authors associate with the values of openness, rationalism, and progress. In contrast, the eleven- and fifteen-year-old students who participated in the WIFI (What I Find Important in learning mathematics) survey valued most, teachers' explanations, knowing the times tables, and correctness. The authors argue that students' valuing of such activities is to be understood as culturally and historically valued actions. They call for caution against the contradiction that democratic inclusion of students' concerns may conserve the values of objectism and control rather than openness and rationalism in mathematics classrooms.

Davis, Carr and Ampadu's work, researching what students value in Ghana, has presented the values research community with relatively rare insights into not just an African perspective, but also that of a country whose students do not perform well in international comparative assessments. The attributes which Ghanaian students value (or not) can help us understand the significance of what are valued (or not) in other countries. The main focus of Davis, Carr and Ampadu's chapter however, is on the potential for students' valuing to change as they transition from primary to junior secondary and then to senior secondary schools. To this end, the questionnaire responses from 1256 Ghanaian primary, junior high school and senior high school students suggest a gradual shift in intensity of student valuing across the school levels, reflecting a greater valuing of all but one of the seven highly valued attributes. Perhaps this shift is in part due to a greater emphasis on high stakes assessment at higher levels of schooling. An exception is the valuing of relevance, which appeared to be most highly valued at the primary school level. The change here can also be explained at least in part with the advent of high stakes assessment at higher levels of schooling. That is, students choosing to study mathematics at the higher secondary school levels might be too preoccupied by the need to perform in examinations that the valuing of mathematics being relevant could be diluted somewhat. On the whole, this study complements the findings of Zhang et al. (2016), which highlights how student values can and do change during the schooling years and provides examples of values change initiatives which are effective.

Hill, Hunter and Hunter explore what middle school Pāsifika students in New Zealand valued most for their mathematics learning in order to contribute more equitable and effective instruction. The authors found that the most important values espoused by the Pāsifika students were utility, peer collaboration/groupwork, effect/practice, and family/familial support. Among these, the values of peer collaboration/group-work and family/familial support were identified as specific to the Pāsifika students. The authors argue that culturally responsive mathematics experiences have the potential to produce more equal learning outcomes for target students. As such, this chapter suggests that for equitable mathematics teaching we need to develop the classroom culture and pedagogy in a better way to align with the mathematics educational values of minority students. 
Kalogeropoulos and Clarkson focus on the value alignment strategies that four Australian teachers used to enhance student engagement when critical incidents arose in the flow of mathematics teaching. Four such value alignment strategies were identified as Scaffolding, Balancing, Intervention and Refuge. These strategies supported the dynamic and flexible nature of value priorities through the ongoing social interaction in the mathematics classrooms. The authors then explore the notion of mathematical identity, which also affects engagement in mathematics learning. Given the complex interplay between values and identity, this chapter suggests that mathematical identity be considered in the alignment of values between a teacher and students.

Abdullah and Leung's chapter demonstrates the relevance of educational values in the consideration of lesson study cycles across cultures. They highlight an earlier observation by Fujii (2014) that the failure for successful replications of the Japanese lesson study model outside Japan could be attributed to a corresponding failure for relevant educational values embedded in the Japanese model to be recognised and represented in the overseas adaptations. They specifically report on a lesson study in a Brunei primary school and found that the values espoused by the teachers reflected local cultural factors (such as the bilingual learning context), which in turn affected the form in which lesson study format took in Brunei.

Dede's chapter reminds us that not only are comparative studies useful with which to study values, but the examination of values across cultures and cultural groups will also help us conduct better comparative studies. Here, Dede drew on the analysis of interviews he conducted with 35 Grade 9 students comprising of German students in Germany, Turkish students in Turkey, and Turkish immigrant students in Germany. Values unique to each group of students were identified. At the same time, it was also observed that the Turkish immigrant students in Germany were not valuing fun in mathematics learning, which highlights how a student's personal experiences might possibly affect how s/he views/regards school education. Dede also identified two values that were common to all three groups of students, namely, rationalism and relevance. That these two values had also been identified by students in other studies elsewhere (as cited by Dede) highlights their pan-cultural significance in mathematics learning.

Nakawa discusses the possibility of incorporating the framework of mathematical, social, and personal values into a number activity. Her qualitative analysis showed that Japanese kindergarten children regarded equality and fairness as very important among their personal and social values. Interestingly these social and personal values became a driving force leading to mathematical values. She suggests that given appropriate situations, social and personal values can serve as a catalyst for quite young children to organically develop mathematical values.

In another study set in Japan, Baba and Shimada are concerned with the notion that mathematics is perceived by students as being value free. They use socially open-ended problems to explore both social and mathematical values with primary aged students and show with such problems, teachers can explore with their students the linkages between the two. Interestingly when students considered the social implications and values inherent in a game-playing situation, they generated various 
mathematical models and then argued that their consideration of the inherent social values concerning the players of the game became a reason for choosing a particular mathematical model. This in itself is an interesting outcome in that so often the mathematical is privileged and other considerations, including the social values of the context, become subservient. Nevertheless the authors argue that the mathematical models that students formulated had to conform to the rationality of mathematics and they found that having students enter into dialog helps this. Indeed students were willing to modify their decisions on both mathematical and social values if they were convinced by the arguments proffered by their peers.

Zhang's chapter takes the reader to mainland China and reports on a comparison of primary and secondary students' valuing. As have other studies reported in this book, he also found that as students progress through years of schooling so their valuing also changes. He also noted that there were some differences attributed to gender. But most interesting was the insight that these students seem to prefer a teacher led approach to teaching, but at the same time a student-centred learning classroom. Such a result would seem strange if reported from western classrooms where these two often are deemed to be in opposition. Results such as these demand deeper thought be given regarding the cultural influences on both teachers' and students' valuing.

Chan and Wong provide an overview on their own previous investigations of students' and teachers' beliefs and values about mathematics, mathematics learning and teaching. The main focus of such an overview was to highlight various research methodologies employed in the studies such as open-ended questions, episode writing, hypothetical situations, mind maps, a variety of interviews (e.g., clinical, semistructured, or stimulus-recalled), quotes from famous mathematicians, classroom observation, snapshots of critical moments, teachers' journals, and questionnaires. The authors argue for the use of hypothetical situations in which the participants need to make a choice under a dilemmatic or extreme situation, because such situations force them to reveal their values. The use of hypothetical situations is promoted to be a complementary methodology in value research to other frequently used ones such as questionnaire and interviews.

The book ends with another study from Turkey. Aktaş, Yakıc1-Topbaş and Dede report on the values that in part played a role in teachers' decision making as they were teaching about polygons in primary schools. As did Bishop and Whitfield (1972) many years ago, the authors note that teachers make decisions before, during and after a lesson. All are important but in different ways, and each set of decisions can be influenced by values (see also Borko et al. 2008). However this study focuses more on the moments during the act of teaching when critical decisions are made and which students notice. These are analyzed and the authors draw out a list of the values they noted in the lessons, that they then set into a frame to show possible inter relationships. 


\subsection{Beginning}

This chapter is just a beginning and it facilitates an opening to the following chapters. Hence we use the continuous form for this section heading. This notion of the continuous is important in both writing and reading. In writing we may well start with a traditional format of knowing what we want to say and have a good idea of the beginning and where and how we want to finish. But in between strange things can happen. To our amazement in writing, a new emerges and the world as we know it changes. In some way we write and discover new ideas and notions about our selves, and so we grow. And it can be the same in reading: We can start by reading a chapter, perhaps because we think it will say something important about ideas we have already formulated, but it does not always work that way. At times, and we hope this happens through the agency of this volume, we end up in quite unexpected places.

This book features the intellectual work of researchers from different ethnicities and nationalities. We hope it will be thought provoking and will be stimulating for you the reader just as much as it has been for us the editors when we put this volume together. As well we hope that the volume will provide an impetus for future conversations about mathematics and values and valuing as we struggle with the immense and ongoing controversies and challenges to make sense of this area, which will bring further insights to researchers, teachers and the wider community.

\section{References}

Bishop, A. (1988). Mathematical enculturation: A cultural perspective on mathematics education. Dordrecht: Springer.

Bishop, A. J., \& Whitfield, R. (1972). Situations in teaching. London: McGraw Hill.

Bishop, A. J. (1996). How should mathematics teaching in modern societies relate to cultural values-some preliminary questions. In Paper presented at the seventh southeast asian conference on mathematics education. Hanoi, Vietnam.

Borko, H., Roberts, S., \& Shavelson, R. (2008). Teachers' decision making: From Alan Bishop to today. In P. Clarkson \& N. Presmeg (Eds.), Critical issues in mathematics education: Major contributions of Alan Bishop (pp. 37-67). Dordrecht: Springer.

Clarkson, P. (2015). Discussion Group report: Connections between valuing and values; rethinking data generating methods. PME Newsletter 7-10.

Clarkson, P., Andersson, A., Bishop, A., Kalogeropoulos, P., \& Seah, W. T. (2017). Connections between valuing and values: Exploring experience and rethinking data generating methods. In G. Kaiser (Ed.), Proceedings of the 13th International Congress on Mathematical Education ICME-13 (pp. 643-644). New York: Springer.

Clarkson, P., \& Presmeg, N. (Eds.). (2008). Critical issues in mathematics education: Major contributions of Alan Bishop. Dordrecht: Springer.

Fujii, T. (2014). Implementing Japanese lesson study in foreign countries: Misconceptions revealed. Mathematics Teacher Education and Development, 16(1), 65-83.

Kaiser, G. (Ed.), (2017). Proceedings of the 13th International Congress on Mathematical Education ICME-13. New York: Springer. 
Seah, W. T., Andersson, A., Bishop, A., \& Clarkson, P. (2016). What would the mathematics curriculum look like if values were the focus? For the Learning of Mathematics, 36(1), 14-20.

Seah, W. T., \& Wong, N.Y. (Eds.). (2012). Values in East Asian mathematics education - The third wave (Special edition). ZDM - The International Journal on Mathematics Education, 44.

Zhang, Q., Barkatsas, A., Law, H., Leu, Y., Seah, W., \& Wong, N. (2016). What primary students in the Chinese mainland, Hong Kong and Taiwan value in mathematics learning: A comparative analysis. International Journal of Science and Mathematics Education, 14(5), 907-924.

Open Access This chapter is licensed under the terms of the Creative Commons Attribution 4.0 International License (http://creativecommons.org/licenses/by/4.0/), which permits use, sharing, adaptation, distribution and reproduction in any medium or format, as long as you give appropriate credit to the original author(s) and the source, provide a link to the Creative Commons license and indicate if changes were made.

The images or other third party material in this chapter are included in the chapter's Creative Commons license, unless indicated otherwise in a credit line to the material. If material is not included in the chapter's Creative Commons license and your intended use is not permitted by statutory regulation or exceeds the permitted use, you will need to obtain permission directly from the copyright holder.

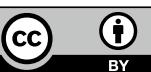

